\title{
Conserved lysine 79 is important for activity of ecto-nucleoside triphosphate diphosphohydrolase 3 (NTPDase3)
}

\author{
Saswata Basu ${ }^{1}$, Deirdre M. Murphy-Piedmonte ${ }^{1,2} \&$ Terence L. Kirley ${ }^{1}$ \\ ${ }^{1}$ Department of Pharmacology and Cell Biophysics, University of Cincinnati, Cincinnati, Ohio, USA; ${ }^{2}$ Amgen Inc., \\ Thousand Oaks, California, USA
}

Received 23 August 2004; accepted in revised form 5 October 2004

Key words: CD39L3, ecto-nucleotidase, NTPDase3, nucleoside triphosphate diphosphohydrolase, oligomeric structure, site-directed mutagenesis

\begin{abstract}
Cell membrane-bound ecto-nucleoside triphosphate diphosphohydrolases (NTPDases) are homooligomeric, with native quaternary structure required for maximal enzyme activity. In this study, we mutated lysine 79 in human ecto-nucleoside triphosphate diphosphohydrolase 3 (NTPDase3). The residue corresponding to lysine 79 in NTPDase 3 is conserved in all known cell surface membrane NTPDases (NTPDase1, 2, 3, and 8), but not in the soluble, monomeric NTPDases (NTPDase5 and 6), or in the intracellular, two transmembrane NTPDases (NTPDase4 and 7). This conserved lysine is located between apyrase conserved region 1 (ACR1) and an invariant glycosylation site (N81), in a region previously hypothesized to be important for NTPDase3 oligomeric structure. This lysine residue was mutated to several different amino acids, and all mutants displayed substantially decreased nucleotidase activities. A basic amino acid at this position was found to be important for the increase of nucleotidase activity observed after treatment with the lectin, concanavalin A. After solubilization with Triton X-100, mutants showed little or no decrease in activity, unlike the wild-type enzyme, suggesting that the lysine at this position may be important for maintaining proper folding and for stabilizing the quaternary structure. However, mutation at this site did not result in global changes in tertiary or quaternary structure as measured by Cibacron blue binding, chemical cross linking, and native gel electrophoretic analysis, leaving open the possibility of other mechanisms by which mutation of this conserved lysine residue might decrease enzyme activity.
\end{abstract}

Abbreviations: ACR - apyrase conserved region; BSA-bovine serum albumin; Con A-concanavalin A; DMEMdulbecco's modified eagle medium; DMSO - dimethyl sulfoxide; DSS - disuccinimido suberate; DTT - dithiothreitol; MOPS - 3-[N-morpholino] propane sulfonic acid; NTPDases - nucleoside triphosphate diphosphohydrolases; $\mathrm{P}_{\mathrm{i}}-$ inorganic phosphate; PVDF - polyvinylidene fluoride; TBS - tris buffered saline

\section{Introduction}

The eNTPDases are a family of enzymes that hydrolyze extracellular nucleoside $5^{\prime} \mathrm{di}$ - and triphosphates [1]. The nucleotidase activity of these enzymes is dependent on the presence of divalent cations (usually $\mathrm{Ca}^{2+}$ or $\mathrm{Mg}^{2+}$ ). Four of the six members of the human eNTPDases characterized to date (NTPDase1-4) are integral membrane glycoproteins, with large extracellular domains and two transmembrane domains located near the $\mathrm{N}$ - and C-termini [1]. Three of these four integral membrane eNTPDases (NTPDase1-3) are cell-surface-associated, while NTPDase4 is associated with Golgi membranes [2]. Recently, another cell-surface associ-

Correspondence to: Dr Terence L. Kirley, Department of Pharmacology and Cell Biophysics, University of Cincinnati College of Medicine, 231 Albert Sabin Way, Cincinnati, OH 45267-0575, USA. Tel: +1-513-5582338; Fax: +1-513-558-1169; E-mail: terry.kirley@uc.edu ated member of this family was reported, mouse NTPDase8, which is expressed at the highest level in liver [3].

Glycosylation of asparagine 81 (N81), an invariant putative glycosylation site located near apyrase conserved region 1 in the NTPDases, has been shown to be essential for full enzymatic activity of NTPDase3 [4]. Enzymatic deglycosylation of this site (by the deglycosylation enzyme, peptide $N$-glycosidase-F) was shown to be responsible for the inactivation of the wild-type enzyme [4]. Removal of the glycosylation site at this position was hypothesized to have its effects by decreasing the stability of the native oligomeric structure [4].

A lysine residue has been hypothesized to be involved in the coordination of a metal ion involved in the catalytic pathway of CD39 (NTPDase1 [5]). However, multiple sequence alignments of NTPDases reveal very few conserved lysine residues, and no conserved lysine residues in the putative phosphate binding domains of the NTPDases, apyrase conserved regions 1 and 4 (ACR1 and ACR4 [6]). 
Table 1. Multiple sequence alignments of the extended NTPDase ACR1 region.

\begin{tabular}{|c|c|c|c|}
\hline eNTPDase & Species & Accession no. & Amino acid sequence (ACR1) \\
\hline \multirow[t]{3}{*}{ NTPDase3 } & Human & AF034840 & 62 DAGSSRTTVYVYQWPAEKENNTGVV \\
\hline & Rat & NP_835207 & 62 DAGSSRTTVYVYQWPAE $\overline{\mathbf{K}}$ ENNTGVV \\
\hline & Mouse & AAU13839 & 62 DAGSSRTTVYVYQWPAE $\overline{\mathbf{K}}$ ENNTGVV \\
\hline \multirow[t]{4}{*}{ NTPDase2 } & Human & AAB81013 & 45 DAGSSHTSMF IYKWPAD玄ENDTGIV \\
\hline & Rat & O35795 & 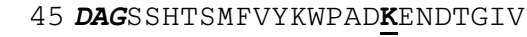 \\
\hline & Mouse & AAC24347 & 45 DAGS SHTSMFVYKWPAD宐ENDTGIV \\
\hline & Chicken & P79784 & 43 DAGS SHTAVF IYKWPAD的ENDTGVV \\
\hline \multirow[t]{4}{*}{ NTPDase1 } & Human & AAB47572 & 54 DAGS SHTSLYIYKWPAE $\overline{\mathbf{K}}$ ENDTGVV \\
\hline & Rat & AAC53195 & 54 DAGS SHTNLYIYKWPAE $\overline{\mathbf{K}}$ ENDTGVV \\
\hline & Mouse & AAH1 1278 & 54 DAGSSHTNLYIYKWPAE $\overline{\mathbf{K}}$ ENDTGVV \\
\hline & Pig & NP_999318 & 54 DAGSSHTSLYIYKWPAE $\overline{\mathbf{K}}$ ENDTGVV \\
\hline NTPDase8 & Mouse & AY3644442 & 48 DAGS SHTSLFVYQWPANKE KDTGVV \\
\hline NTPDase4 & Human & NP_004892 & 94 DCGSSGSRVFVYCWPRHNGNPHDLL \\
\hline NTPDase7 & Human & AAF90135 & 89 DCGSSGSRIFVYFWPRHNGNPHDLL \\
\hline NTPDase5 & Human & AF039918 & 54 DAGSTGTRIHVYTFVQKMPGQLPIL \\
\hline NTPDase6 & Human & AF039916 & 108 DAGSTGTRVHVFQFTRPPRETPTLT \\
\hline
\end{tabular}

The sequence alignment shows the region near apyrase conserved region 1 (ACR1). The first 'DXG' phosphate binding motif is in bold and in italics. The mutated lysine near ACR1 (K79 in NTPDase3, in bold and underlined) is conserved in NTPDases 1, 2, 3, and 8, which are all oligomeric cell surface membrane proteins. This lysine residue is not conserved in NTPDases 4 and 7, which are intracellular membrane proteins, which, like the cell surface NTPDases, also have both N-terminal and C-terminal transmembrane helixes. This lysine residue is also not conserved in NTPDases 5 and 6 , which are associated with intracellular membranes via a single, $\mathrm{N}$-terminal transmembrane domain, and can be secreted as soluble, monomeric proteins after cleavage of the N-terminal signal peptides. Not all known NTPDase sequences are shown in the table, but the rule holds for all sequences currently in the database - lysine at this position is conserved in all cell surface NTPDases (NTPDase 1-3, 8), and not found in all other NTPDases (NTPDase4-7).

Nevertheless, there are two fairly well conserved lysine residues nearby ACR1, Lys 56 and Lys 79 in NTPDase3. In the present study, we analyzed mutations of Lys 79, a conserved lysine residue located nearby the conserved N81 glycosylation site. Lys 79 is conserved in all known sequences of the oligomeric, cell surface NTPDases1, 2, 3 , and 8 , but not in the soluble, monomeric enzymes, NTPDase5 and NTPDase6 (see Table 1). Analyses of the mutants included solubilization (and monomerization) by Triton X-100, native gel electrophoresis, cross-linking by DSS, Cibacron blue binding, and Concanavalin A binding, in addition to nucleotidase assays in the presence of $\mathrm{Ca}^{2+}$ or $\mathrm{Mg}^{2+}$. The results indicate that lysine 79 is essential for full nucleotidase activity of NTPDase 3 , and we speculate this residue might be important for maintaining some subtle aspect of native folding and/or native oligomeric structure necessary for maximal enzymatic activity.

\section{Materials and methods}

\section{Materials}

The QuikChangeTM site-directed mutagenesis kit was purchased from Stratagene. Oligonucleotides were synthesized by the DNA Core Facility at the University of Cincinnati. Lipofectamine Plus Reagent, Dulbecco's modified eagle medium (DMEM), calf serum, and antibiotics/ antimycotics were obtained from Gibco/Life Technologies. The mammalian expression vector pcDNA3 was obtained from Invitrogen. The chemical cross-linking reagent disuccinimidyl suberate (DSS) and the SuperSignal chemiluminescence reagents were purchased from Pierce Chemical. Cibacron Blue Gel (Affi-Gel Blue), pre-cast SDS-PAGE
4\%-15\% gradient mini-gels, and goat anti-rabbit horseradish peroxidase conjugated secondary antibody were obtained from Bio-Rad Laboratories. Ampicillin, nucleotides and other reagents were from Sigma.

\section{Site-directed mutagenesis of NTPDase3}

Mutagenesis of NTPDase3 in pcDNA3 vector was performed using the QuikChange ${ }^{\mathrm{TM}}$ site-directed mutagenesis kit (Stratagene) as described previously [6-11]. The sense nucleotides used for mutagenesis are as follows: K79A, 5'-CAATGGCCAGCAGAAGCAGAGAATAATACCGGAGTGG-3'; K79E， 5'-CAATGGCCAGCAGAAGAA-

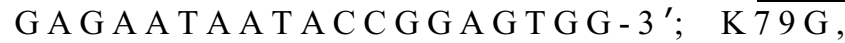
5'-CAATGGCCAGCAGAAGGAGAGAATAATACCGGAGTGG-3'; K79R, 5'-CAATGGCCAGCAGAAAGAGAGAATAATACCGGAGTG-3'; Altered codons are underlined, but the complementary antisense oligonucleotides also necessary for mutagenesis are not shown. DNA sequencing of 600-800 bases surrounding the mutated bases both confirmed the desired mutation and demonstrated that no unintended mutations occurred. The entire coding sequence was not sequenced because our experience in generating more than 100 mutants using this cDNA and methodology have led us to the conclusion that unwanted mutations typically occur within 20-30 bases of the desired mutation. The mutated NTPDase 3 cDNA was used to transform competent cells as described by the manufacturer (Stratagene).

\section{Transient transfection}

COS-1 cells were grown and transfected with wild-type and mutant NTPDase3 cDNA, as previously described [6-11]. An empty pcDNA3 vector was transfected into 
COS cells and used as a background control for nucleotidase assays. Cells were harvested $48 \mathrm{~h}$ post-transfection and crude total membrane preparations were obtained as described [6-11].

\section{Protein assay}

Protein concentrations were determined using the Bio-Rad Coomassie blue dye binding assay, using bovine serum albumin as the standard, with the modifications of Stoscheck [12].

\section{Nucleotidase assays}

Nucleotidase activities were determined by measuring the concentration of inorganic phosphate $\left(\mathrm{P}_{\mathrm{i}}\right)$ released from the ATP or ADP substrates in the presence of $\mathrm{Mg}^{2+}$ or $\mathrm{Ca}^{2+}$. Assays were performed at $37{ }^{\circ} \mathrm{C}$ as previously described [9-11], modified from Fiske and Subbarow [13]. Nucleotidase activities were corrected for COS-1/pcDNA3 background (provided by the empty pcDNA3 vector transfected into COS-1 cells) and for differences in expression levels relative to those of wild-type, as determined by Western blotting.

\section{Western blot analysis}

For Western blot, proteins were resolved in a $4 \%-15 \%$ linear gradient SDS-PAGE gradient gel (BioRad 4\%-15\%) and transferred to a polyvinylidene fluoride (PVDF) membrane. Blots were probed with an anti-C-terminal peptide NTPDase3 antibody, as previously described [6].

\section{Chemical cross-linking}

COS cell membrane preparations $(0.1 \mathrm{mg} / \mathrm{ml})$ were diluted

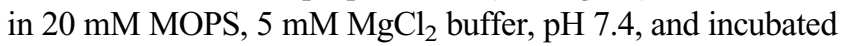
for $20 \mathrm{~min}$ at $22{ }^{\circ} \mathrm{C}$ with $200 \mu \mathrm{M}$ disuccinimido suberate (DSS) freshly dissolved in DMSO. This lysine specific crosslinking agent is able to cross-link NTPDase3 monomers in contact in the native oligomer, and has been used as a probe for oligomeric structure [14]. Non-cross-linked samples were treated with the same volume of DMSO, and the final DMSO concentration of all samples was less than $2 \%$ of the total sample volume. The cross-linking reaction was stopped by incubation with $10 \mathrm{mM}$ lysine for $5 \mathrm{~min}$ at $22{ }^{\circ} \mathrm{C}$. After reducing sample buffer was added, samples were boiled for 5 min, loaded on a SDS-PAGE gradient gel, and Western blotted, as described above.

\section{Analysis of Cibacron blue binding}

Cibacron blue binding assays have been described previously $[7,9,10]$. Basically, this triazine dye binds to many nucleotide-handling enzymes, presumably at the nucleotide binding site. Therefore it has been shown to be a useful probe of the native tertiary structure by demonstrating if various mutations have affected the gross tertiary structure of NTPDase3, thereby affecting the ability of the mutated enzyme to bind the triazine dye.
Native gel electrophoresis of wild-type and mutant NTPDases

NTPDase 3 membrane preparations were solubilized for 10 min at room temperature in digitonin ( $1 \%$ final concentration), a detergent known to preserve the native quaternary structure and the activity of the NTPDases [15, 16]. Solubilized proteins were isolated by centrifugation and electrophoresed on a $6 \%$ Laemmli native gel containing $0.1 \%$ digitonin as described previously [10].

\section{Treatment of wild-type and mutant NTPDase 3 with concanavalin A}

Concanavalin A (Con A) was prepared at a concentration of $5 \mathrm{mg} / \mathrm{ml}$ in $20 \mathrm{mM}$ MOPS buffer containing $100 \mathrm{mM}$ $\mathrm{NaCl}, 1 \mathrm{mM} \mathrm{MnCl}_{2}$ and $1 \mathrm{mM} \mathrm{CaCl} 2, \mathrm{pH}$ 7.4. Wild-type, K79A, K79E, K79G and K79R NTPDase3 total membrane preparations $(2 \mu \mathrm{g})$ were incubated with $5 \mu \mathrm{l}$ of $5.0 \mathrm{mg} / \mathrm{ml}$ Con A at $37{ }^{\circ} \mathrm{C}$ for $15 \mathrm{~min}$ before the addition of substrate. Nucleotidase assays were performed in the presence of $5 \mathrm{mM} \mathrm{Ca}^{2+}$ or $\mathrm{Mg}^{2+}$, at a final nucleotide concentration of $2.5 \mathrm{mM}$.

\section{Time dependence of $M g$-ATPase activity}

Kinetics of $\mathrm{Mg}^{2+}$-dependent ATPase hydrolysis before and after treatment with the lysine-specific cross linker, DSS, were measured in a Beckman DU-800 spectrophotometer, using a NADH-linked enzyme spectrophotometric assay [17], as described previously for NTPDase2 [18] and NTPDase 3 [4]. This assay measures the oxidation of NADH (via the decrease in absorbance at $340 \mathrm{~nm}$ ), which is coupled enzymatically to the hydrolysis of ATP in the presence of $\mathrm{Mg}^{2+}$, allowing continuous measurement of $\mathrm{Mg}^{2+}$-ATPase activity. After pre-incubation to bring the cuvettes and samples to $37^{\circ} \mathrm{C}$, the reactions were initiated by addition of a small volume of NTPDase 3 enzyme.

\section{Solubilization and nucleotidase assays}

Cell membranes $(0.1 \mathrm{mg} / \mathrm{ml})$ were solubilized in $1 \%$ Triton $\mathrm{X}-100,5 \mathrm{mM} \mathrm{MgCl}_{2}$ and $20 \mathrm{mM}$ MOPS buffer $\mathrm{pH} 7.4$ at $22{ }^{\circ} \mathrm{C}$ for $10 \mathrm{~min}$ with occasional mixing, followed by centrifugation at $150,000 \mathrm{~g}$ for $30 \mathrm{~min}$ at $22^{\circ} \mathrm{C} . \mathrm{Mg}^{2+}$ and $\mathrm{Ca}^{2+}$ ATPase and ADPase activities were measured in presence of $0.1 \%$ Triton X-100, after adding nucleotide to a final nucleotide concentration of $0.435 \mathrm{mM}$, using a malachite green phosphate assay [19] to measure nucleotidase activities, because Triton X-100 causes turbidity in the modified Fiske and Subbarow assay [13].

\section{Results}

\section{Expression and characterization of NTPDases}

Mutation of the fairly conserved Lys 56 to Ala did not result in diminished nucleotidase activities (data not shown) and 
Table 2. Nucleotidase activities of wild-type (wt) and mutant NTPDase3 enzymes.

\begin{tabular}{|c|c|c|c|c|c|}
\hline $\begin{array}{l}\text { NTPDase } 3 \\
\text { enzyme }\end{array}$ & $\begin{array}{l}\% \text { wt level } \\
\text { of expression }\end{array}$ & $\begin{array}{l}\text { Normalized } \mathrm{Mg}^{2+} \text {-ATPase } \\
\text { activity }(\% \mathrm{wt})\end{array}$ & $\begin{array}{l}\text { Normalized } \mathrm{Ca}^{2+} \text {-ATPase } \\
\text { activity }(\% \mathrm{wt})\end{array}$ & $\begin{array}{l}\text { Normalized } \mathrm{Mg}^{2+} \text {-ADPase } \\
\text { activity }(\% \mathrm{wt})\end{array}$ & $\begin{array}{l}\text { Normalized } \mathrm{Ca}^{2+} \text {-ADPase } \\
\text { activity }(\% \mathrm{wt})\end{array}$ \\
\hline Wild-type & 100 & $144 \pm 10(100)$ & $413 \pm 35(100)$ & $57 \pm 5(100)$ & $83 \pm 15(100)$ \\
\hline K79A & $68 \pm 5$ & $22 \pm 2 \quad\left(15^{*}\right)$ & $92 \pm 12(22)$ & $16 \pm 4\left(28^{*}\right)$ & $36 \pm 4$ \\
\hline K79E & $50 \pm 7$ & $25 \pm 4 \quad\left(17^{*}\right)$ & $97 \pm 18$ & $15 \pm 2\left(26^{*}\right)$ & $36 \pm 4$ \\
\hline K79G & $60 \pm 4$ & $18 \pm 4 \quad(12 *)$ & $88 \pm 22(21)$ & $7 \pm 2(12 *)$ & $30 \pm 5$ \\
\hline K79R & $74 \pm 8$ & $36 \pm 5 \quad(25)$ & $113 \pm 16(27)$ & $12 \pm 3\left(21^{*}\right)$ & $34 \pm 6 \quad(40)$ \\
\hline
\end{tabular}

Values given represent the means \pm standard deviations of three separate transfections (which were matched to their own wild-type and empty pcDNA3 vector controls). Activities were measured in presence of $5 \mathrm{mM} \mathrm{MgCl} 2$ or $\mathrm{CaCl}_{2}$ at a final concentration of $2.5 \mathrm{mM}$ ATP or ADP. Values were normalized for different NTPDase 3 protein expression levels by dividing the nucleotidase activity (expressed as $\mu \mathrm{mol} \mathrm{Pi} / \mathrm{mg}$ protein/h) by the expression level relative to the wild-type enzyme. Note the differences in the degree of loss of $\mathrm{Mg}^{2+}$-stimulated activity compared to the loss of Ca ${ }^{2+}$ stimulated activity for most of the K79 mutants, especially regarding the ADPase activities. Values for the normalized \% wild-type Mg-nucleotidase activities (in parentheses) that are statistically different $(P \leq 0.05)$ from the corresponding Ca-nucleotidase activities (also in parentheses) are indicated by an asterisk $(*)$.

therefore was not investigated further. Wild-type and K79 mutant NTPDase 3 proteins were transiently expressed in COS cells and expression levels were calculated by quantification of Western blots as previously described [10]. The values presented in Table 2 are the means of three separate transfections, and the specific activities for ATP and ADP of the NTPDase 3 mutants were corrected for variations in expression levels relative to those of the wild-type (see Table 2). ATPase and ADPase nucleotidase activities of all mutants were determined in presence of either $\mathrm{Mg}^{2+}$ or $\mathrm{Ca}^{2+}$. For wild-type NTPDase3, activities for $\mathrm{Mg}^{2+}$ and $\mathrm{Ca}^{2+}$-ATPase were 144 and $413 \mu \mathrm{mol} / \mathrm{mg} / \mathrm{h}$, respectively, while activities for $\mathrm{Mg}^{2+}$ and $\mathrm{Ca}^{2+}$-ADPase were 57 and $83 \mu \mathrm{mol} / \mathrm{mg} / \mathrm{h}$, respectively (Table 2). Interestingly, K79 mutants exhibited different amounts of residual $\mathrm{Ca}^{2+}$ - versus $\mathrm{Mg}^{2+}$-mediated nucleotidase activities. For example, in $\mathrm{Mg}^{2+}$ buffer, K79 NTPDase3 mutants show a 4- to 8-fold decrease in ATPase activity and a 3.5to 8-fold decrease in ADPase activity, while in the presence of $\mathrm{Ca}^{2+}$, K79 mutants show smaller (3.5-5) fold decreases in ATPase activity and approximately threefold decreases in ADPase activity (Table 2). Thus, the K79 mutants showed a larger proportional decrease in $\mathrm{Mg}^{2+}$ stimulated activity compared to $\mathrm{Ca}^{2+}$ stimulated activity. Those differences that are statistically significant $(P \leq 0.05)$ when comparing the \% wild-type activity remaining for $\mathrm{Ca}^{2+}$ versus $\mathrm{Mg}^{2+}$ activities of each mutant are indicated in Table 2 with an asterisk (*).

\section{$\mathrm{Mg}^{2+}$-ATPase kinetics of wild-type and K79A mutant NTPDase3 after cross-linking}

To investigate the effect of the lysine specific cross-linker DSS on $\mathrm{Mg}^{2+}$-ATPase activity of wild-type NTPDase 3 and the K79A lysine mutant, the activity was monitored as a function of time utilizing the linked enzyme assay. Interestingly, DSS treatment increased $\mathrm{Mg}^{2+}$-ATPase activity of the wild-type enzyme (consistent with earlier results [4]), but decreased $\mathrm{Mg}^{2+}$-ATPase activity of the K79A mutant (see Figure 1). Previously, such increases in wild-type NTPDase enzyme activity upon chemical cross- linking have been attributed to stabilization of the native quaternary structure $[4,15]$.

\section{Analysis of the global quaternary structure of the mutants} by native gel electrophoresis and DSS cross-linking

The native gel electrophoretic mobilities of wild-type NTPDase 3 and K79 mutants are indistinguishable, suggesting that the mutants have the same gross oligomeric structure as wild-type NTPDase3 (Figure 2, top panel). In addition, a lysine-specific cross-linking agent, disuccinimido substrate (DSS), was also used to investigate quaternary structure. After reducing SDS-PAGE and Western blotting, both wild-type and mutant NTPDases exist as non-crosslinked monomers in the absence of DSS (data not shown).

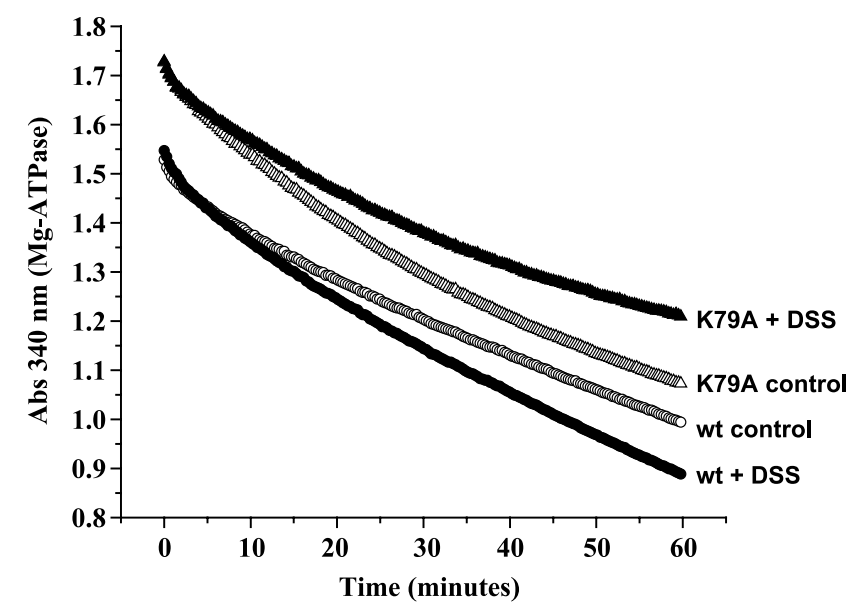

Figure 1. Time dependence of $\mathrm{Mg}^{2+}$-ATPase activity of wild-type and K79A NTPDase 3 after DSS cross-linking. Wild-type and mutant COS cell membranes $(0.1 \mathrm{mg} / \mathrm{ml})$ were treated with DSS $(200 \mu \mathrm{M})$ for $10 \mathrm{~min}$ at room temperature, the reaction was stopped with lysine, and $\mathrm{Mg}^{2+}$ ATPase activity was measured by the NADH-linked enzyme assay [17]. Due to the lower amount of activity of the K79A mutant, $3 \mu \mathrm{g}$ was used for each wild-type sample, while $9 \mu \mathrm{g}$ was used for each K79A NTPDase3 sample, to obtain curves of approximately the same shape, thus allowing easier visual comparison. For clarity of presentation, the data for wild-type NTPDase3 (both control and DSS cross-linked) have been shifted down by subtracting 0.2 absorbance units from each data point. 
Following treatment with $200 \mu \mathrm{M}$ DSS, wild-type and all NTPDase 3 mutants made in this study were similarly crosslinked into dimers, suggesting that the gross quaternary structures of the mutants are similar to those of the wild-type (see Figure 2, middle panel).

Analysis of the global tertiary structure of the mutants by Cibacron blue binding

To explore the possibility of global misfolding induced by point mutations leading to the inability to bind the nucleotide analogue triazine dye, Cibacron blue, Cibacron blue binding assays were performed as previously described [10]. Grossly misfolded mutants are unable to bind Cibacron blue affinity matrix, as is denatured NTPDase 3 (exemplified by the boiled, wild-type enzyme [10]). All the mutants described in this study bound to the Cibacron blue matrix like the wild-type enzyme (see Figure 2, bottom panel), suggesting no global changes in the tertiary conformations of the mutants affecting the ability to bind the nucleotide analogue, Cibacron blue.

\section{Effect of concanavalin A on NTPDase3 wild-type and lysine mutants}

Concanavalin $\mathrm{A}$ is a tetravalent protein that binds to glycans and can stabilize the oligomeric structure of glycoproteins by induction of the formation of protein oligomeric lattices [20]. Concanavalin A was previously
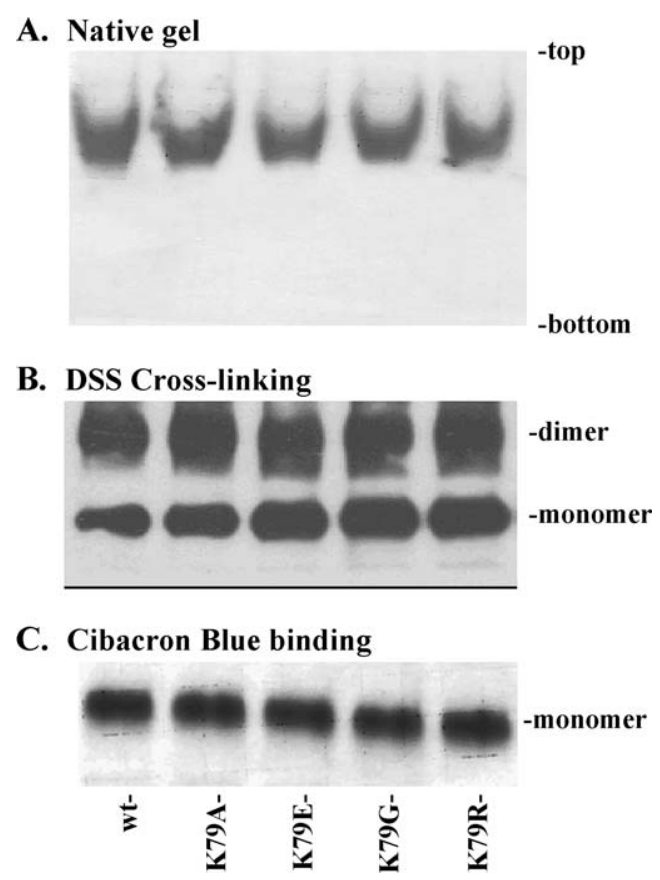

Figure 2. Native gel electrophoresis, DSS cross-linking, and Cibacron blue binding of the NTPDase3 mutants. Native gel electrophoresis (top panel, A) was performed in the presence of $0.1 \%$ digitonin after solubilization of COS cell membranes with $1 \%$ digitonin. DSS crosslinking of membranes (middle panel, B) and analysis of binding of NTPDase3 to Cibacron blue matrix after NP-20 solubilization (bottom panel, C) were performed as described in Materials and methods. All three resultant Western blots were probed with an anti-peptide antibody raised against the C-terminal of the human NTPDase3 [6].

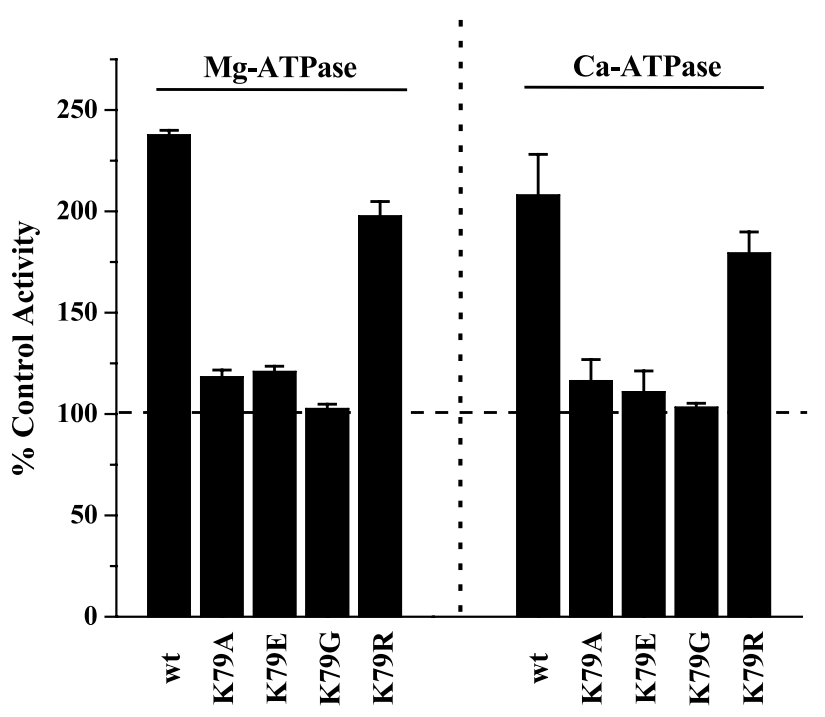

Figure 3. Effect of Concanavalin A on wild-type and K79 mutant NTPDase 3 nucleotidase activities measured in presence of $\mathrm{Mg}^{2+}$ and $\mathrm{Ca}^{2+}$. COS cell membrane preparations expressing wild-type or $\mathrm{K} 79$ mutant NTPDase 3 were incubated with Con A, or control buffer at $37^{\circ} \mathrm{C}$ for $15 \mathrm{~min}$ as described in Materials and methods. Nucleotidase assays were performed in presence of $\mathrm{Ca}^{2+}$ or $\mathrm{Mg}^{2+}$ after initiating the reaction by adding ATP to a final concentration of $2.5 \mathrm{mM}$.

shown to increase the nucleotidase activity of several cell surface, oligomeric NTPDases [4, 18, 21-23]. Unlike wildtype NTPDase 3 and the K79R mutant, which show approximately a 1.9 -fold increase in activity after incubation with Con A, the K79A, K79E, and K79G mutant ATPase activities were not substantially stimulated by Con A (Figure 3). Thus, the nature of the amino acid at this position plays an important role. A basic, positively charged residue at this position is necessary for the stimulatory effect of Con A, while acidic (K79E) or uncharged (K79G and K79A) substitutions at this position greatly diminish this effect. In contrast, the K79R mutant NTPDase $3 \mathrm{Mg}^{2+}$ ATPase activity was decreased by DSS cross-linking (data not shown), similar to the K79A mutant, but dissimilar to the wild-type enzyme shown in Figure 1. Thus, the K79R mutant that responded like wild-type NTPDase3 to Con A treatment (mediated via glycan binding) did not respond like wild-type enzyme to chemical cross-linking by DSS (mediated via reaction at lysine residues).

\section{Do mutations at position 79 affect the NTPDase3 oligomer?}

To investigate whether the effects of mutation of Lys 79 might be dependent on the oligomeric structure, wild-type and K79 mutants were solubilized with Triton X-100. $\mathrm{Ca}^{2+}$ and $\mathrm{Mg}^{2+}$ ATPase activities as well as ADPase activities were determined (Figure 4). Solubilization decreases both $\mathrm{Mg}^{2+}$ and $\mathrm{Ca}^{2+}$-ATPase for wild-type NTPDase3 (Figure 4A). For the wild-type enzyme, Triton X-100 treatment decreased the $\mathrm{Mg}^{2+}$-ATPase activity $75 \%$, while only decreasing the $\mathrm{Ca}^{2+}$-ATPase activity by $37 \%$. $\mathrm{Mg}^{2+}$ ADPase activity of wild-type was decreased by $67 \%$ while $\mathrm{Ca}^{2+}$-ADPase activity was unchanged or increased slightly (Figure 4B). In contrast, for the lysine mutants, the activity 
A

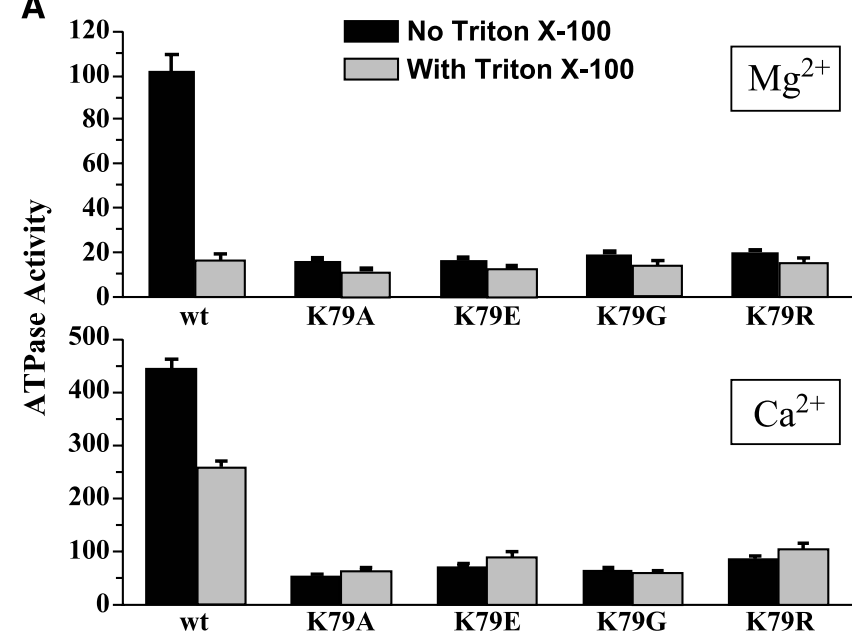

B

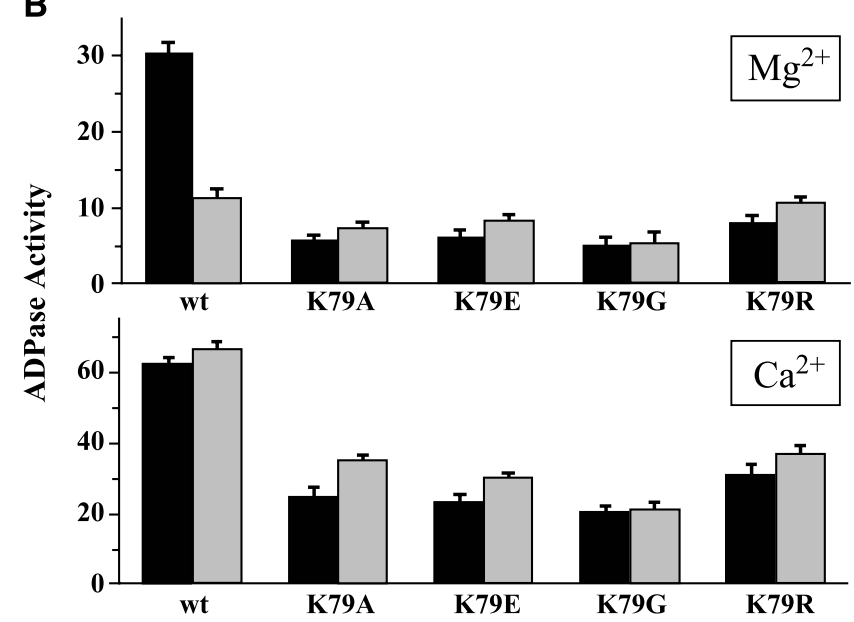

Figure 4. Effect of Triton X-100 on $\mathrm{Ca}^{2+}$ and $\mathrm{Mg}^{2+}$ ATPase and ADPase activities of NTPDase 3 wild-type and K79 mutants. K79A and wild-type COS cell membrane preparations $(0.1 \mathrm{mg} / \mathrm{ml})$ were incubated with $1 \%$ Triton X-100 for $10 \mathrm{~min}$ as described in Materials and methods. Nucleotidase assays were performed in the presence of $\mathrm{Ca}^{2+}$ and $\mathrm{Mg}^{2+}$, at a final ATP concentration of $0.435 \mathrm{mM}$. Panel A (top) is ATPase data, while panel B (bottom) is ADPase data. All activities are reported in units of $\mu \mathrm{mol} / \mathrm{mg} / \mathrm{h}$, and are not corrected for differential expression levels. Values given represent the means \pm standard deviations for three experiments.

remaining after Triton $\mathrm{X}-100$ solubilization is $80 \%$ for $\mathrm{Mg}^{2+}$-ATPase, while there is a $30 \%$ increase in the $\mathrm{Ca}^{2+}$ ATPase activity for all the mutants, except the K79G mutant, whose $\mathrm{Ca}^{2+}$-ATPase activity remains unchanged (Figure 4A). Both the $\mathrm{Mg}^{2+}$-ADPase and $\mathrm{Ca}^{2+}$-ADPase activities of the K79 mutants were increased by $25 \%-30 \%$, except for the $\mathrm{K} 79 \mathrm{G}$ mutant, which remained unchanged (Figure 4B).

\section{Discussion}

In this study we used site-directed mutagenesis to mutate the conserved lysine residue at position 79 to several different amino acids to determine the role of lysine at this position in NTPDase3. This lysine residue is conserved in all cell-membrane associated NTPDases [1-3, 8] sequenced to date, but is not conserved in the soluble
NTPDase 5 and NTPDase 6 enzymes, or in the intracellular membrane associated NTPDase 4 and NTPDase 7 enzymes (see Table 1). This led us to consider the possibility that K79 is important for native oligomeric protein interactions of the cell surface membrane-bound NTPDases.

Continuous measurement of $\mathrm{Mg}^{2+}$-ATPase activity indicates that wild-type NTPDase3 activity increases after cross-linking, in contrast to the $\mathrm{Mg}^{2+}$-ATPase activity of the K79A mutant, which is inhibited after cross-linking (Figure 1). The increase in wild-type activity after crosslinking has been proposed to be the result of intermolecular cross-linking and the resultant stabilization of the native oligomeric structure for both NTPDase3 [4], and other $[15,18]$ membrane-bound NTPDases. Therefore, this result is consistent with the possibility that K79 is important for, or directly involved in, the formation of an inter-molecular cross-link that stabilizes the native quaternary structure, thereby increasing enzyme activity. The observed decrease in K79A activity after cross-linking could be due to (non-cross-linking) modification of essential lysine residues, or to intra-molecular cross-linking, or both. These inhibitory effects of DSS treatment would presumably be masked in the wild-type enzyme by the larger stimulatory effect of inter-molecular crosslinking, possibly involving K79.

Concanavalin A, a tetravalent lectin known to stabilize the oligomeric structure of glycoproteins by induction of high-order protein oligomer lattice formation [20], increases the nucleotidase activity of several eNTPDases [21-27], presumably by modulating the oligomeric structure/stability of the enzyme. Unlike the wild-type enzyme, the K79A, K79E, and K79G NTPDase3 mutants are not stimulated substantially by Con A. However, when lysine is replaced by another basic amino acid (arginine, i.e., the K79R mutant), the residual activity behaves like wild-type with regard to its stimulation by interaction with Con A (see Figure 3), suggesting that a positively charged amino acid at this position is needed for the increase in activity induced by the lectin. However, this K79R mutant does not behave like the wild-type enzyme in that it has significantly lower nucleotidase activity than wild-type enzyme (Table 2), and its activity is not increased by DSS crosslinking (data not shown).

A Cibacron Blue binding assay, which is a measure of gross tertiary structure changes, suggests that all these mutants are not globally misfolded (Figure 2). Chemical cross-linking and native gel electrophoresis results suggest that these mutants also do not undergo global changes in quaternary structure (Figure 2). Thus, the results presented in Figure 2 suggest that the decreases in nucleotidase activity observed for the K79 mutants are not due to global protein misfolding or to gross changes in oligomeric structure.

Triton X-100 solubilization is known to disrupt the oligomeric structure of the cell-surface NTPDases, resulting in monomeric enzymes $[11,16]$. After Triton X-100 solubilization, wild-type NTPDase 3 loses most of its MgATPase activity and there is a substantial decrease in $\mathrm{Ca}^{2+}$ ATPase activity (see Figure 4A). However, solubilization has little effect on the $\mathrm{Mg}^{2+}$-ATPase activity of the K79 
mutants, and $\mathrm{Ca}^{2+}$-ATPase activities of these mutants are slightly increased after solubilization (Figure 4A). One interpretation of these results is that the lysine residue at this position may, in some subtle way, not detectable by DSS cross-linking/Western blot or native gel electrophoresis (Figure 2), stabilizes the quaternary structure of wildtype NTPDase3. The conserved glycosylation site, which is located two residues removed from K79 (at N81), was previously shown to be important for enzyme activity, apparently mediated by its affect on the native quaternary structure [4]. Thus, it is possible that mutation of K79 might affect the local protein conformation and therefore the nearby N81 glycosylation site. Consistent with this hypothesis is the inability of Concanavalin A to increase the enzymatic activity of most of the K79 mutants (Figure 3), as well as the lack of inhibition of the K79 mutants by monomerization with Triton X-100 (Figure 4). Therefore, these results lead us to hypothesize that K79, and this region of the protein in general, may be important in some subtle way for the stability or fine-tuning of the native quaternary structure necessary for maximal enzyme activity. It follows that since the quaternary structures of the K79 mutants are already non-optimal, further disruption of the quaternary structure by Triton X-100 monomerization would have a reduced effect on the residual $\mathrm{Mg}^{2+}$-ATPase activity of these mutants, which is what is observed (see Figure 4). Alternatively, the K79 residue could be directly important for enzyme catalysis. However, this seems not as likely, since this residue is only two amino acids from a known, conserved glycosylation site (N81 in NTPDase3), which should be solvent-accessible, and not in a relatively shielded active site catalytic cleft typical of enzymes. In addition, K79 is not likely to be directly involved in a generic NTPDase active site, since it is not conserved in either the soluble or the intracellular NTPDases (Table 1). Also, mutation of an active site residue would likely diminish $\mathrm{Mg}^{2+}$ - and $\mathrm{Ca}^{2+}$-supported nucleotidase activities to roughly the same extent, which is not the case for the K79 mutants (Table 2).

Although the membrane-bound activities of the wild-type NTPDase 3 and many of the mutant NTPDase 3 constructs (made in this and previous studies) are higher in $\mathrm{Ca}^{2+}$ than in $\mathrm{Mg}^{2+}$, the reason for this is unclear. Based on the differences observed in the $\mathrm{Ca}^{2+}$ versus $\mathrm{Mg}^{2+}$ nucleotidase activities (Figure 4), we speculate that $\mathrm{Ca}^{2+}$ stabilizes the tertiary and quaternary structures of the NTPDase 3 relative to $\mathrm{Mg}^{2+}$, giving rise to a more stable native oligomeric structure and therefore a higher nucleotidase activity. This effect is seen with the enzyme expressed in COS cell membranes, and is even more apparent after solubilization of wild-type NTPDase3 with Triton X-100 (see Figure 4), a detergent that disrupts the oligomeric structure of NTPDases, including NTPDase1/CD39 [16] and NTPDase3 [11]. Thus, the \% decrease of the ATPase activity after solubilization/monomerization of NTPDase3 is greater when assayed using $\mathrm{Mg}^{2+}$-ATP as substrate as opposed to $\mathrm{Ca}^{2+}$-ATP as substrate. After Triton X-100 solubilization, the remaining $\mathrm{Mg}^{2+}$-ATPase activities of the K79 NTPDase3 mutants are virtually the same as the wild- type enzyme, unlike the results obtained in $\mathrm{Ca}^{2+}$ buffer (see Figure 4). This suggests that $\mathrm{Ca}^{2+}$ may have a stabilizing affect on the monomeric tertiary structure, or that $\mathrm{Ca}^{2+}-$ ATP is a better substrate than $\mathrm{Mg}^{2+}$-ATP for the solubilized, monomeric NTPDase3.

In summary, conserved lysine 79, located near ACR1, is essential for maximal NTPDase3 nucleotidase activity. Due to its location very near a known glycosylation site (N81), it seems unlikely that it is directly involved in the active site. Con A and DSS effects on K79 NTPDase3 mutant nucleotidase activities, as well as the effects of Triton X-100 on nucleotidase activities, are consistent with K79 being important for native quaternary structure. However, Cibacron blue binding, native gel electrophoresis, and DSS cross-linking of NTPDase 3 protein indicate no gross changes in tertiary or quaternary structure for the K79 mutants. This suggests that the changes in tertiary or quaternary structure in these mutants must be subtle, and not able to be detected by these techniques. Future application of more sensitive and/or more discriminating techniques to adequately measure small changes in tertiary and quaternary structures may definitively answer the question of the mechanism of loss of activity induced by mutation of lysine 79 .

\section{Acknowledgements}

This work was supported by NIH grants HL59915 and HL72882 to T.L.K.

\section{References}

1. Zimmermann H, Beaudoin AR, Bollen $\mathrm{M}$ et al. Proposed nomenclature for two novel nucleotide hydrolyzing enzyme families expressed on the cell surface. In Vanduffel L (ed): Second International Workshop on Ecto-ATPases and Related Ectonucleotidases, Maastricht, The Netherlands, 1999-2000. Diepenbeek, Belgium: Shaker Publishing 1999; 1-9.

2. Wang T-F, Guidotti G. Golgi localization and functional expression of human uridine diphosphatase. J Biol Chem 1998; 273(18): 11392-9.

3. Bigonnesse F, Levesque SA, Kukulski F et al. Cloning and characterization of mouse nucleoside triphosphate diphosphohydrolase-8. Biochemistry 2004; 43(18): 5511-9.

4. Murphy DM, Kirley TL. Asparagine 81, An invariant glycosylation site near apyrase conserved region 1 , is essential for full enzymatic activity of ecto nucleoside triphosphate diphosphohydrolase 3 . Arch Biochem Biophys 2003; 413(1): 107-15.

5. Chen W, Guidotti G. The metal coordination of sCD39 during ATP hydrolysis. BMC Biochem 2001; 2(1): 9.

6. Smith TM, Kirley TL. Site-directed mutagenesis of a human brain ecto-apyrase: Evidence that the E-type ATPases are related to the actin/heat shock 70/sugar kinase superfamily. Biochemistry 1999; 38(1): 321-8.

7. Smith TM, Lewis Carl SA, Kirley TL. Mutagenesis of two conserved tryptophan residues of the E-type ATPases: Inactivation and conversion of an ecto-apyrase to an ecto-NTPase. Biochemistry 1999; 38 : 5849-57.

8. Hicks-Berger CA, Yang F, Smith TM, Kirley TL. The importance of histidine residues in human ecto-nucleoside triphosphate diphoshohy- 
drolase-3 as determined by site-directed mutagenesis. Biochim Biophys Acta 2001; 1547: 72-81.

9. Yang F, Hicks-Berger CA, Smith TM, Kirley TL. Site-directed mutagenesis of human nucleoside triphosphate diphosphohydrolase 3: The importance of residues in the apyrase conserved regions. Biochemistry 2001; 40(13): 3943-50.

10. Kirley TL, Yang F, Ivanenkov VV. Site-directed mutagenesis of human nucleoside triphosphate diphosphohydrolase 3: The importance of conserved glycine residues and the identification of additional conserved protein motifs in eNTPDases. Arch Biochem Biophys 2001; 395(1): 94-102.

11. Murphy DM, Ivanenkov VV, Kirley TL. Identification of cysteine residues responsible for oxidative cross-linking and chemical inhibition of human nucleoside triphosphate diphosphohydrolase 3. J Biol Chem 2002; 277: 6162-9.

12. Stoscheck CM. Increased uniformity in the response of the Coomassie blue $\mathrm{G}$ protein assay to different proteins. Anal Biochem 1990; 184: 111-6.

13. Fiske $\mathrm{CH}$, Subbarow $\mathrm{Y}$. The colorimetric determination of phosphorous. J Biol Chem 1925; 66: 375-400.

14. Smith TM, Kirley TL. Glycosylation is essential for functional expression of a human brain ecto-apyrase. Biochemistry 1999; 38(5): 1509-16.

15. Stout JG, Kirley TL. Control of cell membrane ecto-ATPase by oligomerization state: Intermolecular cross-linking modulates ATPase activity. Biochemistry 1996; 35(25): 8289-98.

16. Wang T-F, Ou Y, Guidotti G. The transmembrane domains of ectoapyrase (CD39) affect its enzymatic activity and quaternary structure. J Biol Chem 1998; 273(38): 24814-21.

17. Schwartz A, Allen JC, Haragaya S. Possible involvement of cardiac $\mathrm{Na}^{+}, \mathrm{K}^{+}$-ATPase in the mechanism of action of cardiac glycosides. J Pharmacol Exp Ther 1969; 168: 31-41.
18. Hicks-Berger CA, Kirley TL. Expression and characterization of human ecto-ATPase and chimeras with CD39 ecto-apyrase. IUBMB Life 2000; 50: 43-50.

19. Baykov AA, Evtushenko OA, Avaeva SM. A malachite green procedure for orthophosphate determination and its use in alkaline phosphatase-based enzyme immunoassay. Anal Biochem 1988; 171(2): 266-70.

20. Mandal DK, Brewer CF. Interactions of concanavalin A with glycoproteins: Formation of homogenous glycoprotein-lectin crosslinked complexes in mixed precipitation systems. Biochemistry 1992; 31: 12602-9.

21. Moulton MP, Sabbadini RA, Norton KC, Dahms AS. Studies on the transverse tubule Mg-ATPase - lectin induced alterations of kinetic behavior. J Biol Chem 1986; 261: 12244-51.

22. Megias A, Martinez-Senac MM, Delgado J, Saborido A. Regulation of transverse tubule ecto-ATPase activity in chicken skeletal muscle. Biochem J 2001; 353: 521-9.

23. Stout JG, Kirley TL. Purification and characterization of the ectoMg-ATPase of chicken gizzard smooth muscle. J Biochem Biophys Methods 1994; 29(1): 61-75.

24. Beeler T, Gable KS, Keffer JM. Characterization of the membrane bound $\mathrm{Mg}^{2+}$-ATPase of rat skeletal muscle. Biochim Biophys Acta 1983; 734: 221-34.

25. Kirley TL, Gerber LK, Smith TM. Expression and characterization of chicken muscle ecto-ATPase in mammalian COS cells. IUBMB Life 1999; 48: 67-72.

26. Caldwell CC, Hornyak SC, Pendleton E et al. Regulation of chicken gizzard ecto-ATPase activity by modulators that affect its oligomerization status. Arch Biochem Biophys 2001; 387(1): 107-16.

27. Marcus AJ, Broekman MJ, Drosopoulos JH et al. The endothelial cell ecto-ADPase responsible for inhibition of platelet function is CD39. J Clin Invest 1997; 99(6): 1351-60. 